\title{
Silencing of MBD1 reverses pancreatic cancer therapy resistance through inhibition of DNA damage repair
}

\author{
JIN XU ${ }^{1-3 *}$, WENWEI ZHU ${ }^{1-3 *}$, WENYAN XU ${ }^{1-3 *}$, XIAOBO CUI ${ }^{4}$, LEON CHEN $^{4}$, SHUNRONG JI $^{1-3}, \mathrm{YI} \mathrm{QIN}^{1-3}$, \\ WANTONG YAO ${ }^{1-3}$, LIANG LIU ${ }^{1-3}$, CHEN LIU ${ }^{1-3}$, JIANG LONG $^{1-3}$, MIN LI $^{1-4}$ and XIANJUN YU ${ }^{1-3}$ \\ ${ }^{1}$ Department of Pancreatic and Hepatobiliary Surgery, Fudan University Shanghai Cancer Center; \\ ${ }^{2}$ Department of Oncology, Shanghai Medical College, Fudan University; ${ }^{3}$ Pancreatic Cancer Institute, \\ Fudan University, Shanghai 200032, P.R. China; ${ }^{4}$ The Vivian L. Smith Department of Neurosurgery, \\ The University of Texas Medical School at Houston, Houston, TX 77030, USA
}

Received January 18, 2013; Accepted February 22, 2013

DOI: $10.3892 /$ ijo.2013.1901

\begin{abstract}
High resistance to traditional chemo- and radiotherapies contributes to the poor prognosis of pancreatic cancer (PC). Methyl-CpG binding domain protein 1 (MBD1), which plays an important role in disease progression, contributes to the drug resistance of PC cells; however, the mechanism underlying the drug resistance endowed by MBD1 remains unknown. In this study, we found that MBD1 was recruited to DNA damage sites under DNA damage conditions. Silencing of MBD1 significantly impaired activation of the DNA damage checkpoint response and inhibited DNA repair capacity. MBD1 binds mediator of DNA damage checkpoint protein 1 (MDC1), which is induced by radiation and regulates NBS1 activation in the presence of DNA damage repair. Knockdown of MBD1 significantly increased the sensitivity of cells to radiation and cisplatin (diamindichloridoplatin, DDP) in vitro. Importantly, the function of MBD1 in regulating chemoradioresistance is also partially dependent on DNA damage repair. Thus, we hypothesize that MBD1 may promote PC chemoradioresistance by regulating $\mathrm{PC}$ cell fate in the presence of DNA damage. Collectively, these findings reveal an important function of MBD1 in DNA repair and mediation of chemoradioresistance of cancer cells. Moreover, this study suggests that MBD1 is a promising
\end{abstract}

Correspondence to: Dr Xianjun Yu, Department of Pancreatic and Hepatobiliary Surgery, Fudan University Shanghai Cancer Center, No. 270, Dong'An Road, Xuhui District, Shanghai 200032, P.R. China E-mail: yuxianjun88@hotmail.com

Dr Min Li, The Vivian L. Smith Department of Neurosurgery, The University of Texas Medical School at Houston, 6431 Fannin Street, MSE R266, Houston, TX 77030, USA

E-mail: min.li@uth.tmc.edu

*Contributed equally

Key words: pancreatic cancer, methyl-CpG binding domain protein 1, DNA damage repair, chemoradiotherapy resistance molecular target for sensitizing resistant PC tumor cells to chemoradiotherapy.

\section{Introduction}

Pancreatic cancer (PC) is currently the leading cause of cancerrelated mortality in western countries and China (1). Despite improvements in medical treatment for this cancer, the prognosis for PC patients is still very poor. Resistance to chemo- and radiotherapy is very common and directly contributes to the poor outcomes of PC patients (2). This resistance is thought to stem from both the intrinsic nature of PC cells (3) and the abundant fibrotic stroma of the tumors, which favors rapid tumor progression and creates a physical barrier to prevent drug delivery and immune cell infiltration $(4,5)$. The mechanism of this chemoradioresistance, however, remains to be elucidated. Thus, it is of the utmost clinical importance to determine the molecular characteristics underlying this resistance and to identify effective strategies to overcome the resistance.

The main mechanism of radiotherapy involves its ionization action, which can kill the tumor cell either directly or indirectly through generation of DNA double-strand breaks (DSB) in the cells. Tumor cell death via induction of DNA damage is also a potential mechanism for some chemotherapeutics such as cisplatin (diamindichloridoplatin, DDP) and camptothecin. Thus, precise regulation of the DNA damage response is crucial for cellular survival and can potentially dictate the sensitivity of both chemotherapy and radiotherapy in different cancers (6).

Methyl-CpG binding domain protein 1 (MBD1), which binds to methylated $\mathrm{CpG}$ islands and couples DNA methylation to transcriptional repression (7), has been implicated in gene regulation, chromatin formation and genome stability (8). Our previous study showed that MBD1 plays an important role in silencing tumor suppressor genes in PC cell lines (9). More recently, we found that silencing MBD1 may restore sensitivity to chemotherapy and therefore enhance apoptosis in human PC cell lines, however, the molecular mechanism of MBD1's involvement in therapy resistance of PC cells is not clear. Interestingly, Watanabe et al (10) have reported previously that MBD1 is detached from the methyl-CpG sites under the condi- 
tion of DNA damage. Knockdown MBD1 inhibits repair of the damaged DNA and increases the cell sensitivity to the DNA damage treatment. Therefore, we hypothesize that MBD1 may affect the sensitivity of both chemotherapy and radiotherapy through the DNA damage response in PC.

In this study, we demonstrated that MBD1 was recruited to sites of DNA damage and was involved in DNA damage repair in PC cells. Knockdown of MBD1 in PC cells enhanced DNA damage-induced apoptosis and restored the sensitivity of these cells to chemoradiotherapy, suggesting that MBD1 may be a potential therapeutic target to overcome chemoradiotherapy resistance in PC.

\section{Materials and methods}

Cell culture and chemicals. Human PANC-1 and 293T cells were purchased from Shanghai Institutes for Biological Science (Shanghai, China). Cells were cultured in Dulbecco's modified Eagle's medium (DMEM; Gibco-BRL) supplemented with $10 \%$ fetal bovine serum (FBS; Gibco-BRL) at $37^{\circ} \mathrm{C}$ with $5 \% \mathrm{CO}_{2}$. DMEM, FBS, horse serum, L-glutamine $(2 \mathrm{mM})$, penicillin $(50 \mathrm{IU} / \mathrm{ml})$ and streptomycin $(50 \mu \mathrm{g} / \mathrm{ml})$ were purchased from Life Technologies Inc.

Lentiviral production and infection of PC cells. The lentiviral vector pLKO.1 TRC (Addgene Plasmid 10878) was used according to the manufacturer's instructions (http://www. addgene.org/tools/protocols/plko/). In brief, shRNA oligos targeting human MBD1 (sh-MBD1) or MDC1 (sh-MDC1) were designed and cloned into the pLKO.1-TRC cloning vector digested with EcoRI and AgeI. The recombinant construct was co-transfected together with two packaging vectors psPAX2 and pMD2.G into 293T cells. A pLKO.1-scramble shRNA (sh-CTL; Addgene Plasmid 1864) was used as a negative control. Lentiviral particles were harvested and filtered, and then target PC cells were infected with these lentiviral particles. For overexpression of MBD1 or MDC1, FLAG-tagged MBD1 or HA-tagged MDC1 was cloned into the lentiviral vector pWPI.1. Lentiviral particles were produced by co-transfection of pWPI.1-MBD1-FLAG, psPAX2, and pMD.G into 293T cells.

Irradiation (IR) and clonogenic survival assay. Cell monolayers were grown in vitro and irradiated using $6 \mathrm{MV} \mathrm{X}$-rays from linear accelerators (Elekta Synergy, Stockholm, Sweden) with a single dose of $0,2,4$ or 8 Gy of IR. A standard colony-forming assay was performed to determine the surviving fractions. For the clonogenic survival assay, cells were seeded in 6-well tissue culture dishes. The seeded cell number was increased with the dose of IR as described (11). After defined time periods, cells were fixed with $70 \%$ ethanol and stained with methylene blue. Colonies with $>50$ cells were scored as survivors. Non-irradiated cultures were used for data normalization.

Immunofluorescence microscopy. Cells were seeded onto microscope slides and allowed to adhere overnight. After $24 \mathrm{~h}$, slides were treated with $\mathrm{H}_{2} \mathrm{O}_{2}$ or DDP or irradiated at 8 Gy as indicated and fixed with $4 \%$ paraformaldehyde for $15 \mathrm{~min}$. Cells were permeabilized with $0.2 \%$ Triton X-100/phosphate-buffered saline (PBS)/1\% FBS for $10 \mathrm{~min}$ and blocked with $5 \%$ bovine serum albumin/1\% FBS in PBS. Slides were incubated with a rabbit anti-phosphohistone $\mathrm{H} 2 \mathrm{AX}(\gamma \mathrm{H} 2 \mathrm{AX})$ antibody (Epitomics) overnight at $4^{\circ} \mathrm{C}$. Slides were incubated with secondary Alexa 488-conjugated mouse antibodies (Molecular Probes/Invitrogen). Nuclei were counterstained with 4',6-diamino-2-phenylindole (DAPI) and mounted using Vectashield (Vector Laboratories, Peterborough, UK). Radiation-induced $\gamma \mathrm{H} 2 \mathrm{AX}$ foci were counted in at least 100 cells per sample using a fluorescence microscope (Olympus BX 40) and the Leica Application Suite.

Immunoblot analysis. Cells were exposed to various treatments and harvested for immunoblot analysis as described (9). Samples were immunoblotted using antibodies against MDC1, MBD1 (Santa Cruz Biotechnology), total Chk1, phospho-Chk1 (S345), total Chk2, phospho-Chk2 (S19), NBS1, phospho-NBS1 (S343), cleaved caspase-3 (Cell Signaling Technology), total ATM, phospho-ATM (S1981), $\gamma$ H2AX (Epitomics), tubulin, HA and FLAG (Sigma-Aldrich).

Determination of cell proliferation. Approximately $10^{4}$ cells/well were seeded into a 96-well plate and allowed to adhere overnight. After treatment with DDP for 24-48 h, $10 \mu \mathrm{l}$ thiazolyl blue tetrazolium bromide (Sigma-Aldrich) were added, and cells were incubated at $37^{\circ} \mathrm{C}$ for $2 \mathrm{~h}$. Colorimetric measurement was performed at $450 \mathrm{~nm}$ in a microplate reader (Spectra Max 190, Molecular Devices). Experiments were performed in triplicate and repeated at three different times.

Neutral comet assay. DNA damage repair was measured in PANC-1 cells using the comet assay system (Trevigen) according to the manufacturer's instructions. Comet tail moments were scored using Comet Score software (TriTek).

Apoptosis assay. At $72 \mathrm{~h}$ post-transfection, cells were harvested, washed, resuspended in the staining buffer and analyzed with the Vybrant Apoptosis Assay kit (Invitrogen, Carlsbad,CA). Stained cells were detected with a FACSCalibur system, and data were analyzed with CellQuest software (both from Becton-Dickinson, Mountain View, CA). The Annexin V-positive cells were regarded as apoptotic cells.

Co-immunoprecipitation assays. PANC-1 cells and 293T cells were lysed by brief sonication in co-immunoprecipitation buffer (20 mM Tris pH 8.0, $150 \mathrm{mM} \mathrm{NaCl}, 1 \mathrm{mM}$ EDTA, $0.5 \%$ NP-40 supplemented with protease inhibitors). Lysates were centrifuged for $20 \mathrm{~min}$ at $10,000 \mathrm{x} \mathrm{g}$, and the resulting supernatant was pre-cleared by incubation with immobilized protein $\mathrm{A} / \mathrm{G}$ gel (Pierce) for $1 \mathrm{~h}$ at $4^{\circ} \mathrm{C}$. The pre-cleared supernatant was subjected to overnight immunoprecipitation using the indicated antibodies or control $\operatorname{IgG}$ antibodies at $4^{\circ} \mathrm{C}$. The next day, protein complexes were collected by incubation with $25 \mu \mathrm{limmobilized} \mathrm{protein} \mathrm{A} / \mathrm{G}$ gel for $1 \mathrm{~h}$ at $4^{\circ} \mathrm{C}$. The collected protein complexes were washed four times with co-immunoprecipitation buffer and eluted by boiling in protein sample buffer under reducing conditions. The eluted proteins were resolved by sodium dodecyl sulfate polyacrylamide gel electrophoresis and analyzed by western blotting.

Statistical analysis. Statistical analysis was performed using SPSS software for Windows. All analyses used two-sided 
hypothesis tests. Results were expressed as mean \pm standard deviation. Differences between groups were evaluated using the Student's t-test and one-way analysis of variance. P-values $<0.05$ were considered significant.

\section{Results}

MBD1 rapidly accumulates within DNA damage chromatin. The DNA damage response is characterized by the accumulation of checkpoint and DNA repair proteins on the damaged chromatin (12). To investigate whether MBD1 plays a direct role in the DNA damage response, we examined recruitment of MBD1 to sites of DNA damage. We first treated PC cells (PANC-1) with $\mathrm{H}_{2} \mathrm{O}_{2}$, DDP or IR. Each of these treatments induced substantial DNA damage and increased the number of $\gamma \mathrm{H} 2 \mathrm{AX}$ foci, which plays an important role in the repair of DNA lesions by recruiting DNA damage signaling and repair proteins (13). Using a co-immunoprecipitation assay, we confirmed that FLAG-tagged MBD1 co-precipitated with $\gamma \mathrm{H} 2 \mathrm{AX}$ following treatment with different DNA damaging agents as described above (Fig. 1). Thus, we conclude that MBD1 is one of the factors that assemble at sites of DNA damage after treated with $\mathrm{H}_{2} \mathrm{O}_{2}$, DDP or IR.

Silencing MBD1 impairs DNA damage repair in PANC-1 cells. Given that MBD1 accumulates in the damaged chromatin, we next examined whether MBD1 is involved in DNA damage repair in PC cells. We used a comet assay (14) to directly assess the efficiency of DNA damage repair in MBD1-depleted cells. PANC-1 cells treated with sh-MBD1 or control (CTL) RNAs were exposed to $\mathrm{H}_{2} \mathrm{O}_{2}$ and subjected to a neutral comet analysis $6 \mathrm{~h}$ later. As shown in Fig. 2A and B, we detected a slight increase in the level of damaged DNA in untreated MBD-depleted cells. This implies that spontaneous DNA damage accumulates in the absence of MBD1. Importantly, the level of $\mathrm{H}_{2} \mathrm{O}_{2}$-induced DNA damage remained higher at $6 \mathrm{~h}$ in MBD1 knockdown cells, suggesting that MBD1 promotes proper DNA damage repair (Fig. 2A and 2B). This result was further confirmed by assessing the resolution of phosphorylated histone $\mathrm{H} 2 \mathrm{AX}$ $(\gamma \mathrm{H} 2 \mathrm{AX})$ nuclear foci following IR (15). In PANC-1 cells, few $\gamma \mathrm{H} 2 \mathrm{AX}$ foci were observed in unirradiated cells (both sh-CTLand sh-MBD1-treated cells), and comparable levels of foci were induced in sh-CTL and sh-MBD1-treated cells 30 min after IR at $8 \mathrm{~Gy}$ (Fig. 2C). Importantly, $16 \mathrm{~h}$ after IR, few foci remained in the non-silenced control cells, whereas the number of $\gamma \mathrm{H} 2 \mathrm{AX}$ foci in MBD1-depleted PANC-1 cells persisted at considerably higher levels (Fig. 2C). This difference was statistically significant $(\mathrm{P}<0.01$; Fig. 2D).

MBD1 depletion restores chemoradiosensitivity of PANC-1 cells. Since the DNA damage response is tightly connected to the resistance of both chemotherapy and radiotherapy and the fact that MBD1 is an important regulator in PC DNA damage repair, we next examined whether knockdown of MBD1 restores the sensitivity of PC cells to both chemotherapy and radiotherapy. A colony survival assay revealed that PANC-1sh-MBD1 cells were more sensitive to IR (Fig. 3A) or DDP (Fig. 3B) than control cells (PANC-1sh-CTL). Thus, downregulation of MBD1 resulted in increased sensitivity of PC cells to both chemotherapy and radiotherapy. In addition, flow cytometry analysis
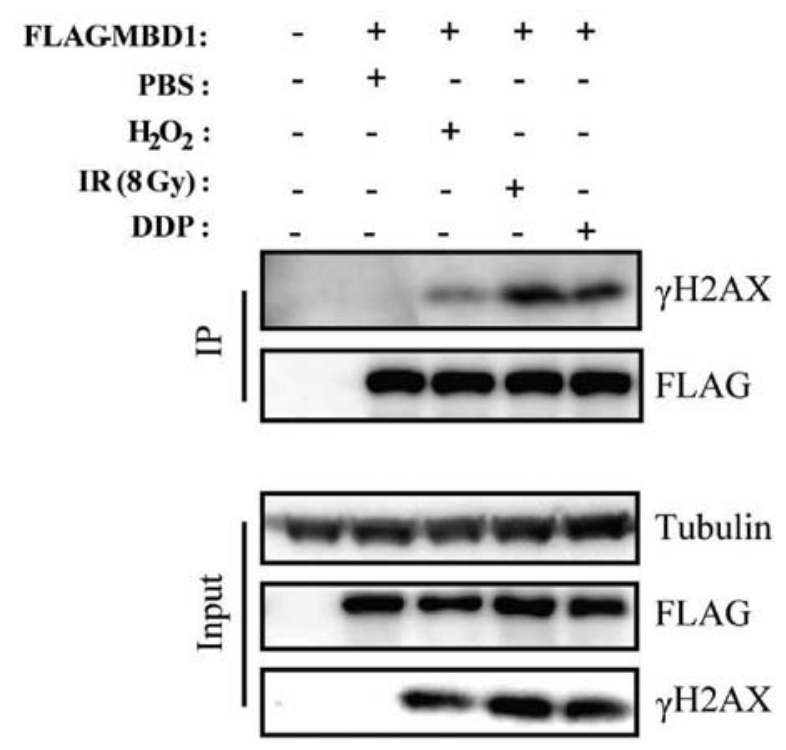

Figure 1. Recruitment of MBD1 to sites of DNA damage. FLAG-tagged MBD1 was transiently expressed in PANC-1 cells, and the whole cell lysates were immunoprecipitated (IP) with anti-FLAG antibody and immunoprecipitated with $\gamma \mathrm{H} 2 \mathrm{AX}$ antibody after treatment with DNA damaging agents $\left(\mathrm{H}_{2} \mathrm{O}_{2}\right.$, DDP and IR).

also confirmed that this increased sensitivity was partially dependent on inducing apoptosis of PC cells (Fig. 3C), as the expression of the pro-apoptotic protein cleaved caspase- 3 was increased in PANC-1sh-MBD1 cells after treatment with $\mathrm{H}_{2} \mathrm{O}_{2}$, IR or DDP compared with PANC-1sh-CTL cells (Fig. 3D).

MBDl activates the DNA damage checkpoint through interaction with MDCl. Although IR and DDP damage tumor cells by way of several mechanisms, these agents kill cancer cells primarily via DNA damage $(6,16)$. Thus, DNA damage checkpoint responses play essential roles in cellular chemoradiosensitivity (17). To determine the role of MBD1 in the DNA damage checkpoint response in PC chemoradioresistance, we examined the DNA damage checkpoint responses in both PANC-1sh-MBDland PANC-1sh-CTL cells after treatment with either IR or DDP. Activating phosphorylation of canonical DNA damage response factors, such as checkpoint protein 1 (pChk1), pChk2, and the checkpoint protein pNBS1 were significantly greater in PANC-1sh-CTL cells than in PANC-1sh-MBD1 cells following exposure to IR or DDP (Fig. 4A and 4B). As MDC1 is known to play an important role in DNA damage response through binding and phosphorylation of NBS1 (18), we hypothesized that the promotion of DNA repair by MBD1 contributes to its interaction with MDC1. The interaction between MBD1 and MDC1 occurred with both overexpressed (Fig. 4C) and endogenous (Fig. 4D) protein, and this interaction was induced by radiation and strengthened with increased exposure to IR treatment (Fig. 4E). Our findings support the notion that inhibition of DNA damage response by silencing MBD1 may be driven in collaboration with effects of MDC1.

$M B D 1$ regulation of chemoradiosensitivity depends on DNA damage repair. Because the DNA damage response was highly correlated with the sensitivity of both chemotherapy and radio- 
A

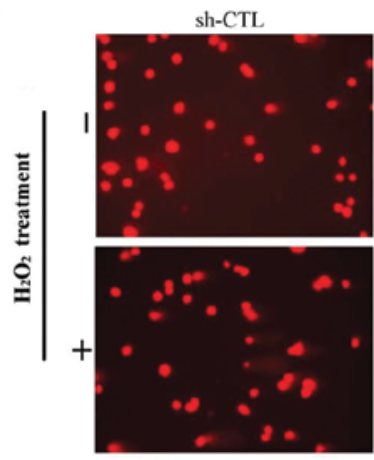

$\mathrm{C}$

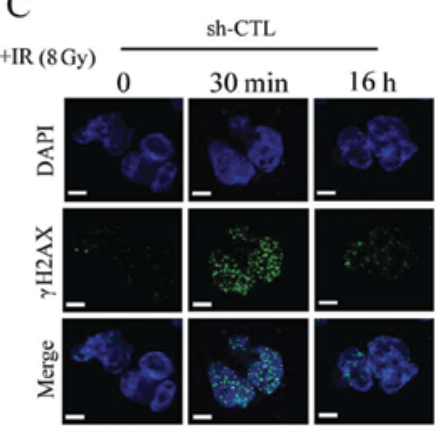

sh-MBD1

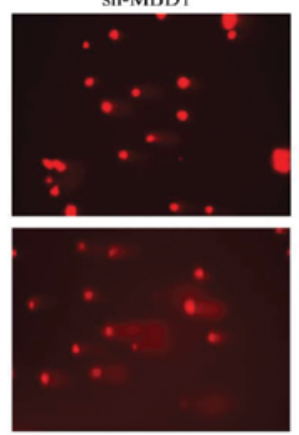

sh-MBDI

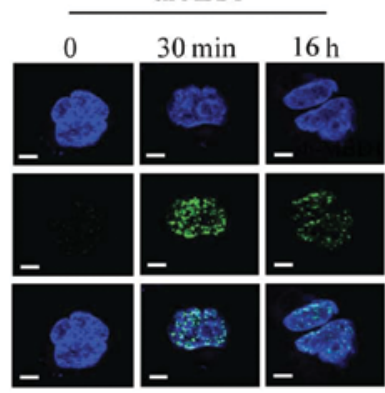

B

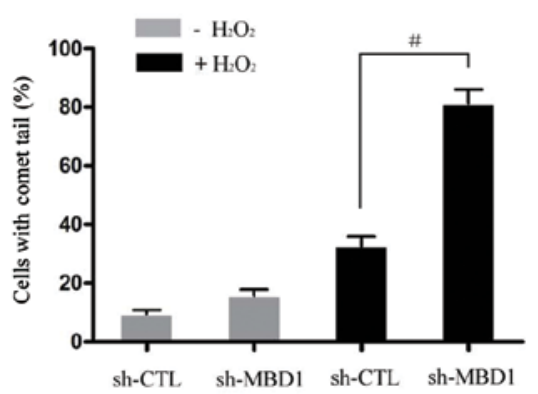

$\mathrm{D}$

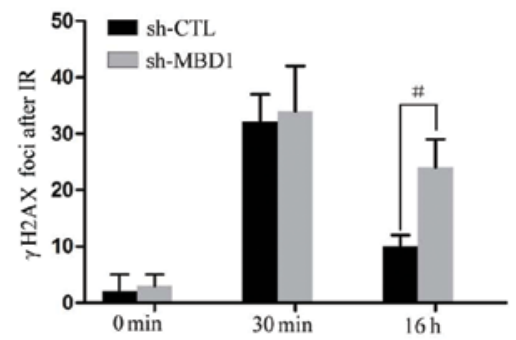

Times after IR (8Gy) treatment

Figure 2. MBD1 depletion impairs DNA damage repair. (A) PANC-1 cells were transfected with the indicated shRNAs and exposed to $\mathrm{H}_{2} \mathrm{O}_{2}$, and subjected to neutral comet analysis after $6 \mathrm{~h}$. Representative images are shown. (B) Quantification of tail moments using cells from (A). Quantification of the percentages of cells with comet tails at the indicated time points after $\mathrm{H}_{2} \mathrm{O}_{2}$ treatment in MBD1-depleted PANC-1 cells (sh-MBD1) and control cells (sh-CTL) are shown (C) Analysis of DNA damage foci formation in PANC-1 cells treated with shRNAs upon exposure to IR at 8 Gy. Immunofluorescence staining for $\gamma \mathrm{H} 2 \mathrm{AX}$ (green) was performed, and nuclei were counterstained with DAPI (blue). Scale bar, $15 \mu \mathrm{m}$. (D) Quantitation of DNA damage foci from (C). Both populations (sh-MBD1 and sh-CTL) showed very few phosphorylated histone $\mathrm{H} 2 \mathrm{AX}(\gamma \mathrm{H} 2 \mathrm{AX})$ foci at the time of radiation and comparable induction of foci 30 min after IR at $8 \mathrm{~Gy}$; however, significantly more $\gamma \mathrm{H} 2 \mathrm{AX}$ foci were observed in sh-MBD1 PANC-1 cells $16 \mathrm{~h}$ after IR than in sh-CTL PANC-1 cells (P<0.01). " Significant difference $(\mathrm{P}<0.01)$.

A

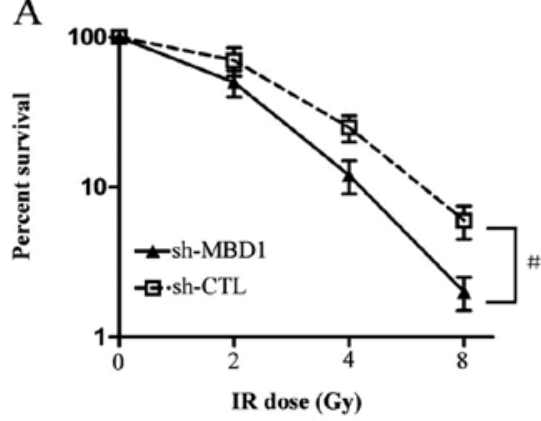

$\mathrm{C}$

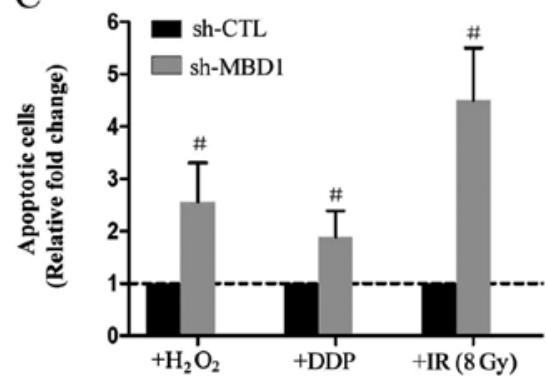

B

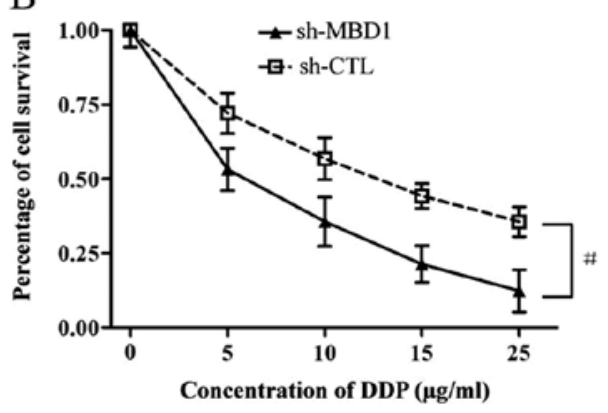

$\mathrm{D}$

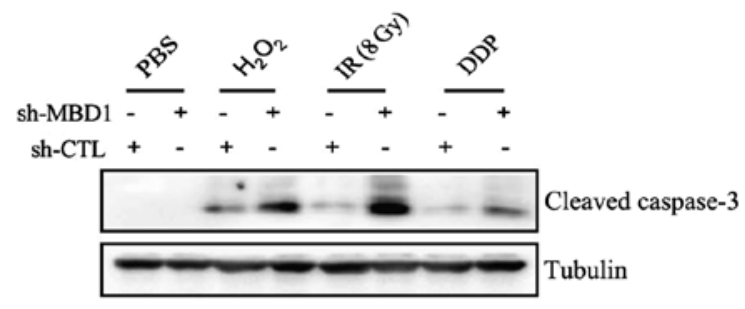

Figure 3. MBD1 depletion restores chemoradiosensitivity of PANC-1 cells. (A) Colony survival assay of PANC-1 cells expressing anti-MBD1 shRNA (sh-MBD1) or control shRNA (sh-CTL) after the indicated doses of IR. "Significant differences $(\mathrm{P}<0.01)$. (B) Downregulation of MBD1 increased chemosensitivity to DDP in PANC-1 cells. PANC-1-sh-MBD1 cells showed increased sensitivity to DDP at different doses. " Significant differences (P<0.01). (C and D) Silencing of MBD1 enhances DNA damage-induced apoptosis. PANC-1 cells expressing anti-MBD1 shRNA (sh-MBD1) or control shRNA (sh-CTL) were treated with $\mathrm{H}_{2} \mathrm{O}_{2}$, DDP or $8 \mathrm{~Gy}$ of IR or left untreated. (C) Relative number of apoptotic cells was determined. "Significant differences ( $\mathrm{P}<0.01)$. (D) Whole-cell lysates were collected after $24 \mathrm{~h}$ and immunoblotted for cleaved caspase-3, an indicator of cell apoptosis. 
A

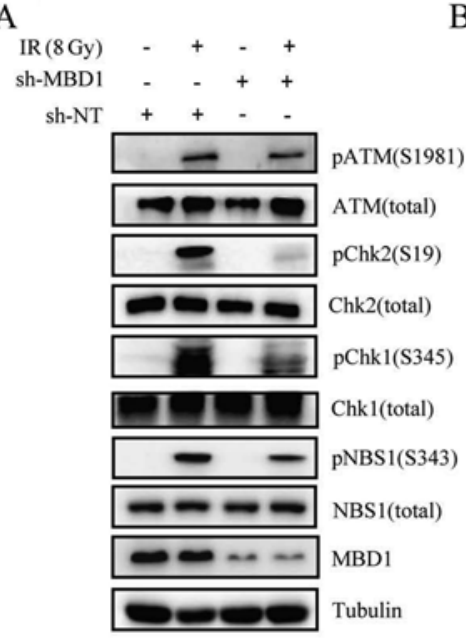

B

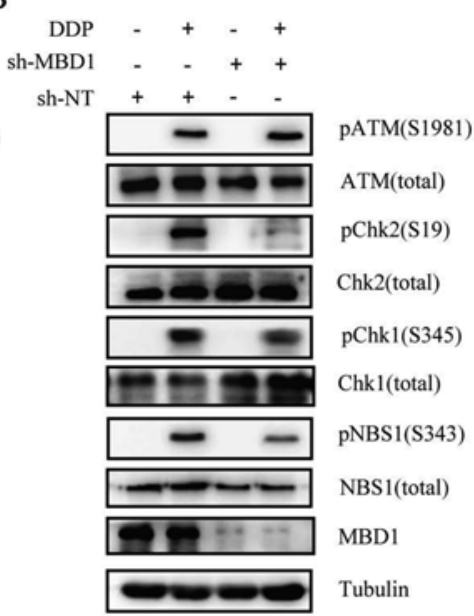

$\mathrm{C}$

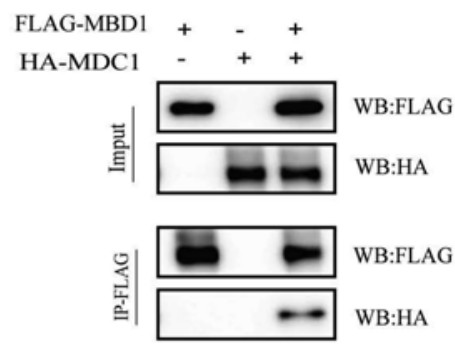

$\mathrm{D}$

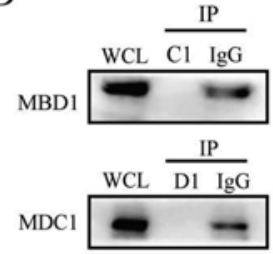

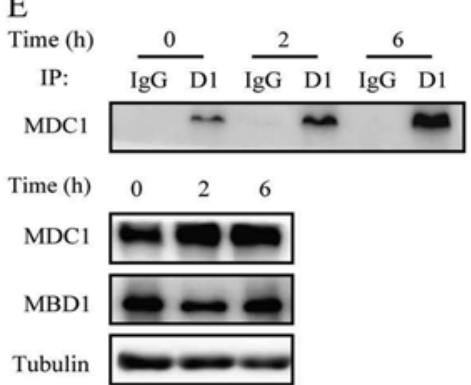

Figure 4. MBD1 interacts with MDC1 to facilitate checkpoint response. (A and B) Knockdown of MBD1 impairs the DNA damage checkpoint response. The activation state of the checkpoint response in matched sh-MBD1 and sh-CTL PANC-1 cells was assessed after treatment with (A) IR or (B) DDP. Whole-cell lysates were immunoblotted for total and phosphorylated checkpoint proteins (ATM, NBS1, Chk2 and Chk1). (C) MBD1 interacts with MDC1. The indicated constructs (FLAG-MBD1 or HA-MDC1) were transiently expressed in 293T cells, and the whole cell lysates were immunoprecipitated (IP) with anti-FLAG antibody and immunoblotted with the relevant antibody. (D) The interaction between endogenous MBD1 and MDC1 in PANC-1 cells as detected by immunoprecipitation-immunoblotting. (E) MBD1-MDC1 interaction at different time points after irradiation of PANC-1 cells. Top panel, MBD1 immunoprecipitation-MDC1 immunoblotting. Lower panel, levels of MBD1 and MDC1 after IR as determined by immunoblot analysis.

therapy, we asked whether restoration of chemoradiotherapy sensitivity in PC was at least partly dependent on the inhibition of DNA damage response by the interaction between MBD1 and MDC1. We introduced MBD1 into cells that had stable silencing of MDC1 via shRNA (PANC-1sh-MDC1) along with an MBD1-expressing plasmid (Fig. 5A). These cells were used in the subsequent experiments to evaluate the degree of inhibition after treatment with chemo- or radiotherapeutic agents (DDP or IR). Cells with MBD1 overexpression were resistant to both radiotherapy (Fig. 5B) and chemotherapy (Fig. 5C), but these cells became sensitive to either radiotherapy or chemotherapy following MDC1 knockdown (Fig. 5B and 5C). Furthermore, MDC1-silenced cells did not display a remarkable increase of chemo- or radio-sensitivity immediately upon reintroduction of MBD1, suggesting that MBD1 may regulate cell fate following DNA damage through an interaction with MDC1 and subsequent acceleration of a downstream check point response. These effects may, therefore, increase DNA repair activity and promote chemoradioresistance (Fig. 5D).

\section{Discussion}

Genomic integrity is critical to organismal survival and is controlled by the DNA damage response network, an elabo- rate signal transduction system that senses DNA damage and recruits appropriate repair factors (19). This global signaling network senses the different types of DNA lesions and coordinates a response that includes activation of transcription, cell cycle control, apoptosis, senescence, and/or DNA repair processes (20). It has been hypothesized that one of the most important determinants of chemoradiotherapy resistance in cancer cells may stem from an overall resistance to DNA damage-induced apoptosis (21). PC remains one of the deadliest of all cancers despite aggressive surgical treatment combined with chemo- and radiotherapy. Chemoradioresistance is the principal cause of treatment failure in PC patients and leads to the poor prognosis for patients with the disease $(22,23)$. Strategies to find candidate targets linking DNA damage repair to chemoradioresistance in order to sensitize PC cells to chemoradiotherapy are well underway.

In this study, we report a novel role for MBD1 in the DNA damage repair network in PC cells. We find that MBD1 is recruited to DNA damage sites under DNA damage conditions. Silencing MBD1 significantly impairs activation of the DNA damage checkpoint response and inhibits DNA repair capacity in PC. Our data support the earlier report that MBD1 is detached away from the damaged methyl-CpG sites and may serve as a sensor for damaged bases during the DNA damage response 
A

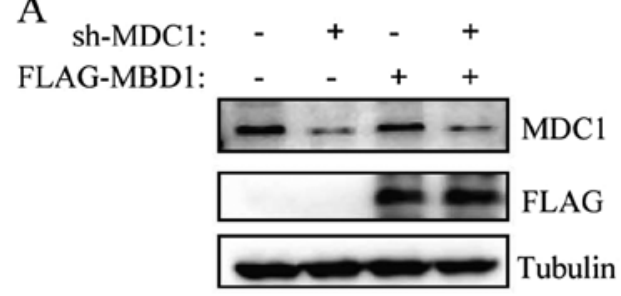

C

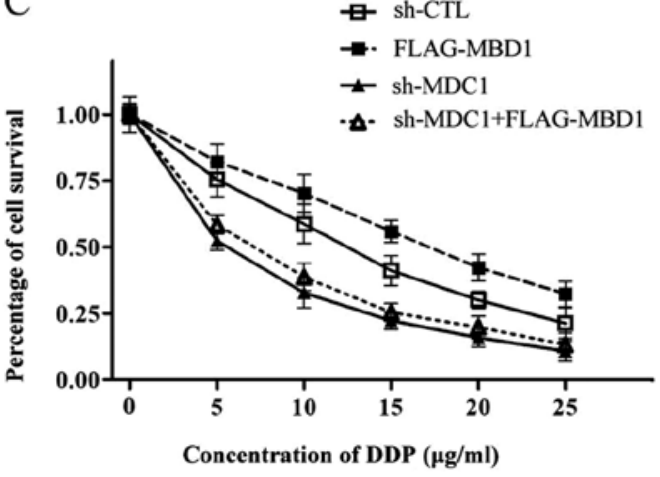

B

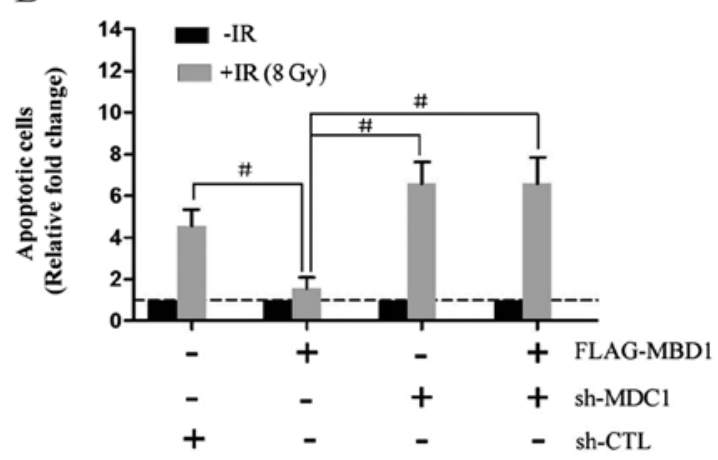

D

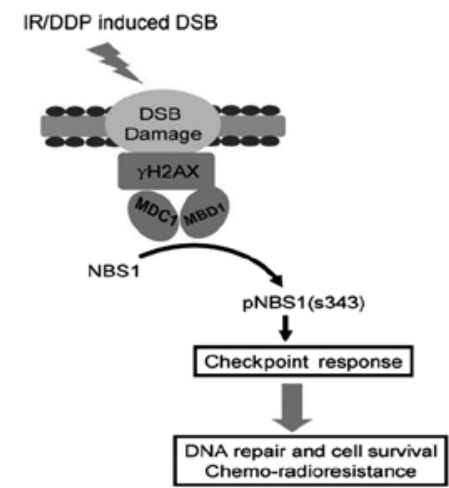

Figure 5. MBD1 regulation of chemoradiosensitivity depends on DNA damage repair. The sh-MDC1-treated PANC-1 cells expressing MBD1 or not (A) were exposed to IR (8 Gy) for apoptosis analysis. Relative cell apoptosis rate was assessed as above. Error bars, SD ( $\mathrm{n}=3$ ). $(\mathrm{B}){ }^{\#}$ Significant differences $(\mathrm{P}<0.01)$. (C) Cells transfected with indicated plasmids (sh-CTL, FLAG-MBD1, sh-MDC1 and sh-MDC1+FLAG-MBD1) were treated with the indicated concentrations of DDP. (D) Proposed model for functional modulation of MBD1 on DNA damage repair and chemoradiosensitivity in PC shows that MBD1 may regulate cell fate in the presence of DNA damage through an interaction with MDC1 to accelerate downstream check point response. This may increase DNA repair activity and promote chemoradioresistance.

to promote chromatin remodeling and repair of the damaged DNA (10), however, the mechanism of MBD1 involvement in DNA damage repair requires further elaboration. Moreover, previous study from our group also demonstrated that the expression levels of pro-apoptosis proteins, such as cleaved caspase-3, -9 and bax, were significantly elevated in MBD1silenced PANC-1 cells after treatment with gemcitabine, which has been reported as an inhibitor of DNA damage repair (24). Taken together, our recent findings together with the previous reports suggest that $\mathrm{MBD} 1$ is involved in DNA damage repair of PC cells and may be a chemosensitizing target for PC treatment.

We also explored the potential mechanism and involvement of MBD1 in regulation of DNA damage repair in PC cells. Knockdown of MBD1 significantly impaired activation of the DNA damage checkpoint response. Following DNA damage, DNA repair and cell cycle checkpoints are the main means of maintaining genomic stability and cell survival (25). Several checkpoints are activated at different stages of the cell cycle. Chk1 is a serine/threonine kinase that is primarily responsible for initiating cell cycle arrest in order to allow adequate time for DNA repair (26), while the serine/threonine kinase Chk2 is activated when phosphorylated by ataxia telangiectasia mutated (ATM) following generation of DNA double-strand breaks. The effects of activated Chk 2 on the effector protein Cdc $25 \mathrm{~A}$ phosphatase are similar to those mediated by Chk1 $(27,28)$. Indeed, changes in MBD1 expression led to alterations in the expression of these cell cycle checkpoint factors in PC cells and these findings further supported our hypothesis that MBD1 affects the DNA damage response through effects on the checkpoint response.

Critical to the recruitment of DNA repair proteins to sites of DNA damage (nuclear foci) is phosphorylation of histone $\mathrm{H} 2 \mathrm{AX}(\gamma \mathrm{H} 2 \mathrm{AX})$ on Ser139 by the protein kinases ATM and ataxia telangiectasia and Rad3-related protein (ATR), which are activated by DNA damage at the core of the DNA damage signaling apparatus (29), leading to the accumulation of repair proteins at the sites of damaged DNA $(30,31)$. Several proteins involved in the DNA damage response contain specific $\mathrm{H} 2 \mathrm{AX}$ recognition domains and $\mathrm{MDC} 1$ is one such protein. A body of evidence indicates that the interaction between MDC1 and $\mathrm{H} 2 \mathrm{AX}$ is the first step in preparing the DNA damage signaling and repair $(26,32)$. We noted in our study that MBD1 was physically binding with both $\gamma \mathrm{H} 2 \mathrm{AX}$ and MDC1 when exposed to DNA damage agents. Importantly, MDC1 has been reported to act upstream of NBS1 and regulate the intra-S-phase checkpoint in response to DNA damage through targeting and activating NBS1 to damage DNA sites directly (33). Considering that knockdown of MBD1 also significantly abrogate NBS1 activation and down-stream checkpoint response following IR or DDP. Thus our results indicated that MBD1 may contribute to the observed chemoradioresistance in PC cells via interference with the DNA damage response in association with MDC1 although further investigation is needed. 
In conclusion, our study provides the first evidence that the methyl-CpG binding domain protein MBD1 is closely involved in mediating the resistance of PC cell lines to chemoradiotherapy. MBD1, therefore, represents a promising molecular target to sensitize a priori-resistant PC to IR. Although these findings have begun to uncover the underlying cellular mechanisms of this chemoradioresistance (Fig. 5D), future studies will ultimately be required to elucidate the mechanism of MBD1 regulation on the DNA damage response. Furthermore, these studies indicated that MBD1 inhibition may be an effective strategy to increase the fraction of patients that respond to multimodal treatment and thus improve overall survival.

\section{Acknowledgements}

This study was partially supported by the National Natural Science Foundation of China(NSFC-30901435, NSFC-30972905, NSFC-81172276 and NSFC-81001058) and Joint Project for Emerging Frontier Technology of Shanghai Hospitals of Municipal Level (SHDC12010120).

\section{References}

1. Siegel R, Ward E, Brawley O and Jemal A: Cancer statistics, 2011: the impact of eliminating socioeconomic and racial disparities on premature cancer deaths. CA Cancer J Clin 61: 212-236, 2011

2. Erkan M, Hausmann S, Michalski CW, et al: The role of stroma in pancreatic cancer: diagnostic and therapeutic implications. Nat Rev Gastroenterol Hepatol 9: 454-467, 2012.

3. Wong HH and Lemoine NR: Pancreatic cancer: molecular pathogenesis and new therapeutic targets. Nat Rev Gastroenterol Hepatol 6: 412-422, 2009.

4. Olive KP, Jacobetz MA, Davidson CJ, et al: Inhibition of Hedgehog signaling enhances delivery of chemotherapy in a mouse model of pancreatic cancer. Science 324: 1457-1461, 2009

5. Garrido-Laguna I, Uson M, Rajeshkumar NV, et al: Tumor engraftment in nude mice and enrichment in stroma-related gene pathways predict poor survival and resistance to gemcitabine in patients with pancreatic cancer. Clin Cancer Res 17: 5793-5800, 2011.

6. Terry SY and Vallis KA: Relationship between chromatin structure and sensitivity to molecularly targeted auger electron radiation therapy. Int J Radiat Oncol Biol Phys 83: 1298-1305, 2012.

7. $\mathrm{Ng} \mathrm{HH}$, Jeppesen $\mathrm{P}$ and Bird A: Active repression of methylated genes by the chromosomal protein MBD1. Mol Cell Biol 20: 1394-1406, 2000.

8. Lopez-Serra L, Ballestar E, Fraga MF, Alaminos M, Setien F and Esteller M: A profile of methyl-CpG binding domain protein occupancy of hypermethylated promoter $\mathrm{CpG}$ islands of tumor suppressor genes in human cancer. Cancer Res 66: 8342-8346, 2006

9. Liu C, Chen Y, Yu X, et al: Proteomic analysis of differential proteins in pancreatic carcinomas: effects of MBD1 knockdown by stable RNA interference. BMC Cancer 8: 121, 2008.

10. Watanabe S, Ichimura T, Fujita N, et al: Methylated DNA-binding domain 1 and methylpurine-DNA glycosylase link transcriptional repression and DNA repair in chromatin. Proc Natl Acad Sci USA 100: 12859-12864, 2003.

11. Zhang Y, Chen LH, Wang L, Wang HM, Zhang YW and Shi YS: Radiation-inducible PTEN expression radiosensitises hepatocellular carcinoma cells. Int J Radiat Biol 86: 964-974, 2010.

12. Sancar A, Lindsey-Boltz LA, Unsal-Kacmaz K and Linn S: Molecular mechanisms of mammalian DNA repair and the DNA damage checkpoints. Annu Rev Biochem 73: 39-85, 2004.
13. Bekker-Jensen S, Lukas C, Kitagawa R, et al: Spatial organization of the mammalian genome surveillance machinery in response to DNA strand breaks. J Cell Biol 173: 195-206, 2006.

14. Jirawatnotai S, Hu Y, Michowski W, et al: A function for cyclin D1 in DNA repair uncovered by protein interactome analyses in human cancers. Nature 474: 230-234, 2011.

15. Löbrich M, Shibata A, Beucher A, et al: gammaH2AX foci analysis for monitoring DNA double-strand break repair: strengths, limitations and optimization. Cell Cycle 9: 662-669, 2010.

16. Bartkova J, Horejsí Z, Koed K, et al: DNA damage response as a candidate anti-cancer barrier in early human tumorigenesis. Nature 434: 864-870, 2005.

17. Kastan MB and Bartek J: Cell-cycle checkpoints and cancer. Nature 432: 316-323, 2004.

18. Chapman JR and Jackson SP: Phospho-dependent interactions between NBS1 and MDC1 mediate chromatin retention of the MRN complex at sites of DNA damage. EMBO Rep 9: 795-801, 2008.

19. Harper JW and Elledge SJ: The DNA damage response: ten years after. Mol Cell 28: 739-745, 2007.

20. Zhou BB and Elledge SJ: The DNA damage response: putting checkpoints in perspective. Nature 408: 433-439, 2000.

21. Yao W, Yu X, Fang Z, et al: Profilin1 facilitates staurosporinetriggered apoptosis by stabilizing the integrin betal-actin complex in breast cancer cells. J Cell Mol Med 16: 824-835, 2012.

22. Shibamoto Y, Kubota T, Kishii K and Tsujitani M: Radiosensitivity of human pancreatic cancer cells in vitro and in vivo, and the effect of a new hypoxic cell sensitizer, doranidazole. Radiother Oncol 56: 265-270, 2000.

23. Kim MP and Gallick GE: Gemcitabine resistance in pancreatic cancer: picking the key players. Clin Cancer Res 14: 1284-1285, 2008.

24. Parsels LA, Morgan MA, Tanska DM, et al: Gemcitabine sensitization by checkpoint kinase 1 inhibition correlates with inhibition of a Rad51 DNA damage response in pancreatic cancer cells. Mol Cancer Ther 8: 45-54, 2009.

25. Krempler A, Deckbar D, Jeggo PA and Lobrich M: An imperfect G2M checkpoint contributes to chromosome instability following irradiation of S and G2 phase cells. Cell Cycle 6: 1682-1686, 2007

26. Bartek J and Lukas J: Mammalian G1- and S-phase checkpoints in response to DNA damage. Curr Opin Cell Biol 13: 738-747, 2001.

27. Taverna SD, Li H, Ruthenburg AJ, Allis CD and Patel DJ: How chromatin-binding modules interpret histone modifications: lessons from professional pocket pickers. Nat Struct Mol Biol 14: 1025-1040, 2007.

28. Mailand N, Falck J, Lukas C, et al: Rapid destruction of human Cdc25A in response to DNA damage. Science 288: 1425-1429, 2000.

29. Helt CE, Cliby WA, Keng PC, Bambara RA and O'Reilly MA: Ataxia telangiectasia mutated (ATM) and ATM and Rad3-related protein exhibit selective target specificities in response to different forms of DNA damage. J Biol Chem 280: 1186-1192, 2005.

30. Falck J, Mailand N, Syljuasen RG, Bartek J and Lukas J: The ATM-Chk2-Cdc25A checkpoint pathway guards against radioresistant DNA synthesis. Nature 410: 842-847, 2001.

31. Kouzarides T: SnapShot: histone-modifying enzymes. Cell 131: $822,2007$.

32. Stucki M, Clapperton JA, Mohammad D, Yaffe MB, Smerdon SJ and Jackson SP: MDC1 directly binds phosphorylated histone $\mathrm{H} 2 \mathrm{AX}$ to regulate cellular responses to DNA double-strand breaks. Cell 123: 1213-1226, 2005.

33. Wu L, Luo K, Lou Z and Chen J: MDC1 regulates intra-S-phase checkpoint by targeting NBS1 to DNA double-strand breaks. Proc Natl Acad Sci USA 105: 11200-11205, 2008. 